\title{
Delaunay and Diamond Triangulations Contain Spanners of Bounded Degree
}

\author{
Prosenjit Bose* $\quad$ Michiel Smid* Daming $\mathrm{Xu}^{*}$
}

\begin{abstract}
Given a triangulation $G$, whose vertex set $V$ is a set of $n$ points in the plane, and given a real number $\gamma$ with $0<\gamma<\pi$, we design an $O(n)$-time algorithm that constructs a connected subgraph $G^{\prime}$ of $G$ with vertex set $V$ whose maximum degree is at most $14+\lceil 2 \pi / \gamma\rceil$. If $G$ is the Delaunay triangulation of $V$, and $\gamma=2 \pi / 3$, we show that $G^{\prime}$ is a $t$-spanner of $V$ (for some constant $t$ ) with maximum degree at most 17 , thereby improving the previously best known degree bound of 23 . If $G$ is a triangulation satisfying the diamond property, then for a specific range of values of $\gamma$ dependent on the angle of the diamonds, we show that $G^{\prime}$ is a $t$-spanner of $V$ (for some constant $t$ ) whose maximum degree is bounded by a constant dependent on $\gamma$. If $G$ is the graph consisting of all Delaunay edges of length at most 1 , and $\gamma=\pi / 3$, we show that a modified version of the algorithm produces a plane subgraph $G^{\prime}$ of the unit-disk graph which is a $t$-spanner (for some constant $t$ ) of the unit-disk graph of $V$, whose maximum degree is at most 20 , thereby improving the previously best known degree bound of 25 .
\end{abstract}

\section{Introduction}

Let $V$ be a set of $n$ points in the plane and let $t \geq 1$ be a real number. An undirected graph $G$ with vertex set $V$ is called a t-spanner of $V$, if for any two vertices $u$ and $v$ of $V$, $G$ contains a path between $u$ and $v$, whose length is at most $t|u v|$, where $|u v|$ denotes the Euclidean distance between $u$ and $v$. The stretch factor (or dilation) of $G$ is defined to be the smallest value of $t$ for which $G$ is a $t$-spanner of $V$.

The problem of constructing a $t$-spanner with $O(n)$ edges for any given point set has been studied intensively; see the book by Narasimhan and Smid [10] for a survey.

In this paper, we focus on spanners that are plane, i.e., the interiors of any two (straightline) edges of the spanner are disjoint. Chew [5] and Dobkin et al. [7] were the first to show the existence of plane spanners. Dobkin et al. proved that the Delaunay triangulation of $V$

*This research was supported by the Natural Sciences and Engineering Research Council of Canada. School of Computer Science, Carleton University, 1125 Colonel By Drive, Ottawa, ON, Canada, K1S 5B6, \{jit, michiel, dxu5\}@scs.carleton.ca 


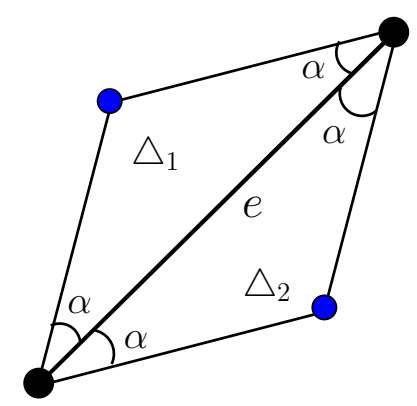

Figure 1: An illustration of the diamond property. At least one of the triangles $\Delta_{1}$ and $\Delta_{2}$ does not contain any point of $V$.

is a $t$-spanner of $V$, for $t=((1+\sqrt{5}) / 2) \pi$. Keil and Gutwin [8] improved the analysis, and showed that the Delaunay triangulation is a $t$-spanner for $t=\frac{4 \pi \sqrt{3}}{9}$. A more general result appears in Bose et al. [3]: For every two vertices $u$ and $v$ of $V$, the Delaunay triangulation contains a path between $u$ and $v$ of length at most $\frac{4 \pi \sqrt{3}}{9} \cdot|u v|$, all of whose edges have length at most $|u v|$.

Das and Joseph [6] generalized these results to triangulations that satisfy the so-called diamond property: Let $G$ be a triangulation of $V$, and let $\alpha$ be a real number with $0<\alpha<\frac{\pi}{2}$. Let $e$ be an edge of $G$, and consider the two isosceles triangles $\Delta_{1}$ and $\Delta_{2}$ with base $e$ and base angle $\alpha$; refer to Figure 1 . We say that the edge $e$ satisfies the $\alpha$-diamond property, if at least one of $\Delta_{1}$ and $\Delta_{2}$ does not contain any point of $V$ in its interior. We say that the triangulation $G$ satisfies the $\alpha$-diamond property, if every edge $e$ of $G$ satisfies this property.

Das and Joseph [6] showed that any triangulation satisfying the $\alpha$-diamond property is a $t$-spanner, for some real number $t$ that only depends on the value of $\alpha$. (In fact, Das and Joseph considered plane graphs that, additionally, satisfy the so-called good polygon property.) The analysis was refined by Bose et al. [2], who showed that $t \leq \frac{8(\pi-\alpha)^{2}}{\alpha^{2} \sin ^{2}(\alpha / 4)}$. The Delaunay triangulation satisfies the $\alpha$-diamond property for $\alpha=\pi / 4$. Das and Joseph proved that both the greedy triangulation and the minimum weight triangulation satisfy the $\alpha$-diamond property, for some constant $\alpha$. The greedy triangulation is formed by looking at all the pairs of points in increasing order of length and selecting an edge as long as it does not cross any edges picked so far. The minimum weight triangulation is a triangulation that minimizes the sum of the lengths of the edges.

None of the results mentioned above leads to plane spanners in which the degree of every vertex is bounded by a constant. For example, the maximum vertex degree in a Delaunay triangulation can be $\Omega(n)$. Bose et al. [1] were the first to show the existence of a plane $t$-spanner (for some constant $t$ ), whose maximum vertex degree is bounded by a constant. To be more precise, they showed that the Delaunay triangulation of any set $V$ of $n$ points in the plane contains a subgraph, which is a $t$-spanner for $V$, where $t=\frac{4 \pi(\pi+1) \sqrt{3}}{9}$, and whose maximum degree is at most 27. This result was improved by Li and Wang [9], who showed that for any real number $\gamma$ with $0<\gamma \leq \pi / 2$, the Delaunay triangulation contains a 
subgraph, which is a $t$-spanner, where $t=\max \left\{\frac{\pi}{2}, 1+\pi \sin \frac{\gamma}{2}\right\} \cdot \frac{4 \pi \sqrt{3}}{9}$, and whose maximum degree is at most $19+\lceil 2 \pi / \gamma\rceil$. For $\gamma=\pi / 2$, the degree bound is 23 . In this paper, we improve the degree bound from 23 as obtained by Li and Wang [9] down to 17. However, the stretch factor is increased approximately to 29 .

Theorem 1. Let $V$ be a set of $n$ points in the plane, and let $\gamma$ be a real number with $0<\gamma \leq 2 \pi / 3$. Assume that we are given the Delaunay triangulation $G$ of $V$. Then, in $O(n)$ time, we can compute a subgraph $G^{\prime}$ of $G$, such that $G^{\prime}$ is a $t$-spanner of $V$, where

$$
t= \begin{cases}\frac{4 \pi \sqrt{3}}{9} \cdot \max \left\{\frac{\pi}{2}, 1+\pi \sin \frac{\gamma}{2}\right\} & \text { if } \gamma<\pi / 2, \\ \frac{4 \pi \sqrt{3}}{9}\left(2+2 \sqrt{3}+3 \pi / 2+2 \pi \sin \frac{\pi}{12}\right) & \text { if } \pi / 2 \leq \gamma \leq 2 \pi / 3\end{cases}
$$

and the maximum degree of $G^{\prime}$ is at most $14+\lceil 2 \pi / \gamma\rceil$. Thus, for $\gamma=2 \pi / 3$, the degree bound is 17 .

We obtain this result by designing a linear-time algorithm that, when given an arbitrary triangulation $G$ of the point set $V$, computes a subgraph $G^{\prime}$ of $G$, that satisfies the degree bound in Theorem 1. We then show that when $G$ is the Delaunay triangulation, the stretch factor of $G^{\prime}$ is at most the value of $t$ in Theorem 1 .

We also extend this result to the unit-disk graph, which is the graph where every two distinct points $u$ and $v$ in the vertex set are connected by an edge if and only if $|u v| \leq 1$. A $t$-spanner of the unit-disk graph is a subgraph of the unit-disk graph with the property that for every edge $(u, v)$ of the unit-disk graph, there exists a path between $u$ and $v$ in the subgraph whose length is at most $t|u v|$. Let $G$ be the graph consisting of all edges in the Delaunay triangulation of $V$, whose length is at most one. It follows from the result of Bose et al. [3] which was mentioned above, that $G$ is a $\frac{4 \pi \sqrt{3}}{9}$-spanner of the unit-disk graph of $V$. The construction for the Delaunay triangulation by Bose et al. [1] was modified by Li and Wang [9] to obtain a plane $t$-spanner (for some constant $t$ ) of the unit-disk graph whose maximum degree is at most 25. By modifying our algorithm, we improve the degree bound from 25 as obtained by Li and Wang [9] down to 20.

Theorem 2. Let $V$ be a set of $n$ points in the plane, and let $\gamma$ be a real number with $0<\gamma \leq \pi / 3$. Assume that we are given the Delaunay triangulation $G$ of $V$. Then, in $O(n)$ time, we can compute a plane graph $G^{\prime}$, such that $G^{\prime}$ is a $t$-spanner of the unit-disk graph of $V$, where

$$
t=\frac{4 \pi \sqrt{3}}{9} \cdot \max \left\{\frac{\pi}{2}, 1+\pi \sin \frac{\gamma}{2}\right\}
$$

and the maximum degree of $G^{\prime}$ is at most $14+\lceil 2 \pi / \gamma\rceil$. Thus, for $\gamma=\pi / 3$, the degree bound is 20 .

We emphasize that the graph $G^{\prime}$ in Theorem 2 is not necessarily a subgraph of the Delaunay triangulation, but it is a plane subgraph of the unit-disk graph.

Bounded degree plane spanners of the unit-disk graph have applications in topology control of wireless ad hoc networks, see [9]. In such a network, a vertex $u$ can only communicate 
with those vertices that are within the communication range of $u$. If we assume that this range is equal to one for each vertex, then the unit-disk graph models a wireless ad hoc network. Many algorithms for routing messages in such a network require that the underlying network topology is plane. Moreover, if the maximum degree is small, then the throughput of the network can be improved significantly.

In the final part of the paper, we show that the algorithm in Theorem 1 can in fact be applied to any triangulation satisfying the diamond property:

Theorem 3. Let $V$ be a set of $n$ points in the plane, let $\alpha$ be a real number with $0<\alpha \leq \pi / 2$, and assume that we are given a triangulation $G$ of $V$ that satisfies the $\alpha$-diamond property. Then, in $O(n)$ time, we can compute a subgraph $G^{\prime}$ of $G$, such that $G^{\prime}$ is a $t$-spanner of $V$, where

$$
t=\left(1+\frac{2(\pi-\alpha)}{\alpha \sin \frac{\alpha}{4}} \cdot \max \left\{1,2 \sin \frac{\alpha}{2}\right\}\right) \frac{8(\pi-\alpha)^{2}}{\alpha^{2} \sin ^{2} \frac{\alpha}{4}}
$$

and the maximum degree of $G^{\prime}$ is at most $14+\lceil 2 \pi / \alpha\rceil$.

Thus, by combining Theorem 3 with the results of Das and Joseph [6], it follows that both the greedy triangulation and the minimum weight triangulation contain a $t$-spanner (for some constant $t$ ) whose maximum degree is bounded by a constant.

The rest of the paper is organized as follows. In Section 2, we present the linear-time algorithm that computes a bounded degree subgraph of any given triangulation. In Section 3 and Section 4, we prove Theorems 1 and 2, respectively, whereas the proof of Theorem 3 is presented in Section 5. We conclude in Section 6 with some directions for future work.

\section{Computing a bounded-degree subgraph of a trian- gulation}

Let $V$ be a set of $n$ points in the plane, and let $G=(V, E)$ be a planar graph. We define a numbering of the elements of $V$ in the following way: pick a vertex of minimum degree and assign it label 1. Then delete this vertex together with its incident edges, increment the label by 1 and recursively define a numbering of the remaining $n-1$ vertices. The resulting numbering $\left(v_{1}, v_{2}, \ldots, v_{n}\right)$ of the vertex set $V$ is called a low-degree numbering. The following lemma explains this terminology and presents an important property of a low-degree numbering.

Lemma 1. Let $v_{i}$ be a vertex in a low-degree numbering of $V$ with index $i, 1 \leq i \leq n$. The number of edges $\left(v_{i}, v_{j}\right)$ of $G$, with $j>i$, is at most 5. Given the planar graph $G, a$ low-degree numbering of $V$ can be computed in $O(n)$ time.

Proof. The first claim follows from the fact that every planar graph contains a vertex of degree at most 5 .

A low-degree numbering can be computed by using an array $A[1 \ldots n]$, where $A[j]$ contains a list of all nodes having degree $j$. With each vertex $w$, we store a pointer to the occurrence of $w$ in the list $A[j]$. 
In the $i$-th iteration of the algorithm, we pick the first vertex $u$ in the subarray $A[1 \ldots 5]$ and label it $v_{i}$. Then we consider all edges $(u, w)$ in the current graph. For any such edge $(u, w)$, if $w$ has degree $j$, then we delete $w$ from the list $A[j]$, and insert it into list $A[j-1]$. Finally, we delete $u$ from the subarray $A[1 \cdots 5]$. Since each node $w$ will be updated at most $\operatorname{deg}(w)$ times and each update takes constant time, the total running time is proportional to $\sum_{w} \operatorname{deg}(w) \leq 2(3 n-6)=O(n)$.

The algorithm that computes a bounded-degree subgraph of any given triangulation is given in Figure 2; for an illustration, refer to Figure 3. In this algorithm, $N_{G}(v)$ denotes the set of neighbors of the vertex $v$ in $G$, i.e. $N_{G}(v)=\{w \in V:(v, w) \in E\}$.

We assume that the triangulation is stored in a doubly-connected edge list. Then, for any vertex $v$, we can obtain the vertices in $N_{G}(v)$, sorted in angular order around $v$, in time proportional to the degree of $v$. Assuming $\gamma$ is constant, this observation, together with Lemma 1 and the fact that $G$ contains $O(n)$ edges, implies that the running time of algorithm BDEGSUBGRAPH is $O(n)$.

Lemma 2. Let $G=(V, E)$ be a triangulation whose vertex set $V$ is a set of $n$ points in the plane, and let $\gamma$ be a real number with $0<\gamma<\pi$. Let $G^{\prime}$ be the graph that is returned by algorithm BDeGSubgraph $(G, \gamma)$. Then, $G^{\prime}$ is a connected subgraph of $G$ with vertex set $V$.

Proof. $G^{\prime}=\left(V, E^{\prime}\right)$ is a subgraph of $G$ with vertex set $V$ because the only edges added to $E^{\prime}$ are from $E$. Therefore, it suffices to show that for each edge $(u, v)$ of $E \backslash E^{\prime}$, the graph $G^{\prime}$ contains a path between $u$ and $v$. Let $(u, v)$ be such an edge of $E \backslash E^{\prime}$. We may assume without loss of generality that $u$ is processed before $v$. Let $C$ be the cone with apex $u$ and having angle at most $\gamma$ that contains $v$ and that is constructed when vertex $u$ is processed. Let $v^{\prime}$ be the closest neighbor of $u$ that is contained in $C$ and that is unprocessed at the moment when $u$ is processed. Thus, during the processing of $u$, the edge $\left(u, v^{\prime}\right)$ is added to $G^{\prime}$. We may assume without loss of generality that $\left(u, v^{\prime}\right)$ is clockwise to the right of $(u, v)$. Observe that $\angle v u v^{\prime} \leq \gamma$.

We distinguish two cases.

Case 1: At the moment when $u$ is processed, all neighbors of $u$ between $v$ and $v^{\prime}$ are unprocessed. Then by steps $14-16$ in algorithm $\operatorname{BDEGSUbGRAPH}(G, \gamma)$, there is a path $P_{v^{\prime} v}$ between $v^{\prime}$ and $v$. Since $\left(u, v^{\prime}\right) \in G^{\prime}$, we have a path from $u$ to $v$ as illustrated in Figure 4(a).

Case 2: At the moment when $u$ is processed, at least one neighbor of $u$ between $v$ and $v^{\prime}$ has been processed. Let $w$ be the last processed neighbor of $u$ in clockwise order of $v$ in $C$, i.e., there is no processed neighbor of $u$ between $v$ and $w$ in $C$. Let $x$ be the first neighbor of $u$ clockwise to the left of $w$ (observe that $x$ could be $v$ ). The vertex $w$ is processed before $x$ and $u$. Since $\Delta_{u w x}$ is a triangle in $G$, when processing $w$, by algorithm $\operatorname{BDEgSubgraph}(G, \gamma)$, edge $(u, x)$ must have been added to $E^{\prime}$. Similar to Case 1 , there is a path $P_{u v}$ between $u$ and $v$ shown as a directed path through vertex $x$ in Figure 4(b). 
Algorithm BDegSubgraph $(G, \gamma)$

Input: A triangulation $G=(V, E)$ whose vertex set $V$ is a set of $n$ points in the plane, and a real number $\gamma$ with $0<\gamma<\pi$.

Output: A subgraph $G^{\prime}=\left(V, E^{\prime}\right)$ of $G$ whose maximum degree is at most $14+\left\lceil\frac{2 \pi}{\gamma}\right\rceil$.

1. compute a low-degree numbering $\left(v_{1}, v_{2}, \cdots, v_{n}\right)$ of $G=(V, E)$;

2. label each vertex of $V$ as "unprocessed";

3. $E^{\prime}=\emptyset$;

4. for $i=n$ downto 1

$5 . \quad$ if $v_{i}$ has "unprocessed" neighbors

6. $\quad$ then compute the closest "unprocessed" neighbor $x$ of $v_{i}$;

7. divide the plane into cones $C_{1}, \ldots, C_{\lceil 2 \pi / \gamma]}$ with apex $v_{i}$ and angle at most $\gamma$; let segment $v_{i} x$ be on the boundary between $C_{1}$ and $C_{2}$;

8. $\quad$ add the edge $\left(v_{i}, x\right)$ to $E^{\prime}$

9. $\quad$ for each cone $C \notin\left\{C_{1}, C_{2}\right\}$

10.

11.

12.

13.

14.

15. do compute the closest "unprocessed" vertex $w$ in $C \cap N_{G}\left(v_{i}\right)$; if $w$ exists

then add the edge $\left(v_{i}, w\right)$ to $E^{\prime}$ let $w_{0}, w_{1}, \cdots, w_{d-1}$ be the clockwise order of the vertices in $N_{G}\left(v_{i}\right)$; for $k=0$ to $d-1$ if $w_{k}$ and $w_{(k+1) \bmod d}$ are both "unprocessed"

16. $\quad$ then add the edge $\left(w_{k}, w_{(k+1) \bmod d}\right)$ to $E^{\prime}$;

17. label $v_{i}$ as "processed";

18. return the graph $G^{\prime}=\left(V, E^{\prime}\right)$

Figure 2: The algorithm that computes a bounded-degree subgraph of a triangulation $G$. 


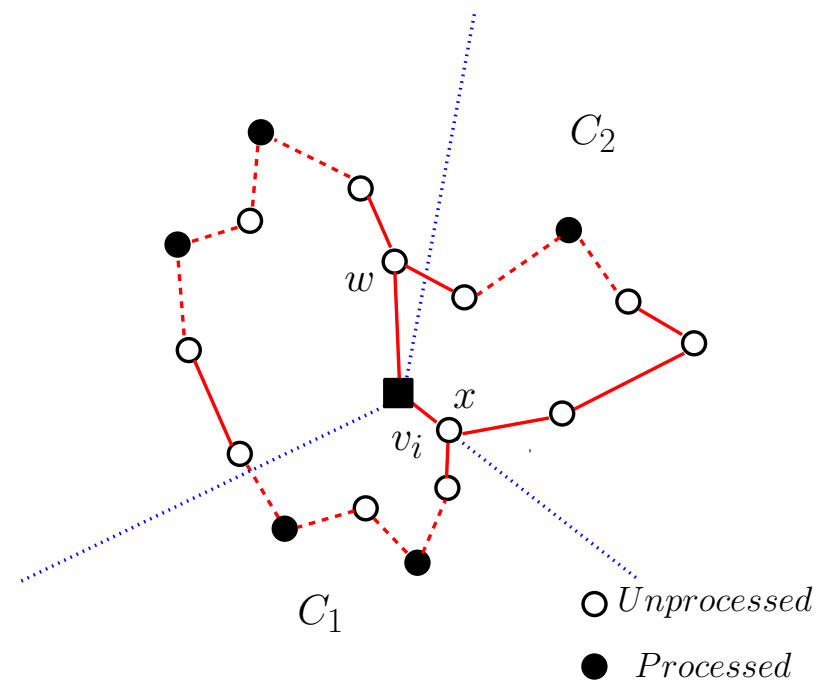

Figure 3: An illustration of algorithm $\operatorname{BDEGSUBGRAPH}(G, \gamma)$, for $\gamma=2 \pi / 3$, when processing vertex $v_{i}$. The figure shows $v_{i}$ and all vertices in $N_{G}\left(v_{i}\right)$. Solid vertices represent the "processed" vertices, whereas hollow vertices represent the "unprocessed" vertices. When processing $v_{i}$, the algorithm adds the solid edges to the graph $G^{\prime}$.

Lemma 3. Let $G$ be a triangulation whose vertex set $V$ is a set of $n$ points in the plane, and let $\gamma$ be a real number with $0<\gamma<\pi$. Let $G^{\prime}$ be the graph that is returned by algorithm $\operatorname{BDegSubgraph}(G, \gamma)$. Then the maximum degree of $G^{\prime}$ is at most $14+\left\lceil\frac{2 \pi}{\gamma}\right\rceil$.

Proof. Let $u$ be an arbitrary element of $V$. Recall that the vertices are processed in reverse order of the low-degree numbering. It follows from Lemma 1 that, before $u$ is processed, it has at most 5 processed neighbors, say $u_{1}, u_{2}, \cdots, u_{k}$, where $k \leq 5$. In the worst case, each of its processed neighbors $u_{j}$ can increase the degree of $u$ by 3 . (This is depicted in Figure 3, where the degree of the unprocessed vertex $w$ is increased by 3 when $v_{i}$ is processed.) This happens when, at the moment that $u_{j}$ is processed, (i) $u$ is the closest unprocessed neighbor of $u_{j}$ in some cone with apex $u_{j}$, and (ii) both neighbors of $u$ adjacent to $u_{j}$ are also unprocessed. Hence, before $u$ is processed, the degree of $u$ (in $G^{\prime}$ ) is at most 15 .

During the processing of $u$, the algorithm divides the plane into $\left\lceil\frac{2 \pi}{\gamma}\right\rceil$ cones. For each cone, the algorithm adds one edge from $u$ to its closest unprocessed neighbor. However, two of these cones $\left(C_{1}\right.$ and $C_{2}$ in the algorithm) share an edge as noted in line 7 of algorithm $\operatorname{BDEgSubgraph}(G, \gamma)$. Thus, during the processing of $u$, the degree of $u$ (in $G^{\prime}$ ) is increased by at most $\left\lceil\frac{2 \pi}{\gamma}\right\rceil-1$.

After $u$ has been processed, the degree of $u$ does not change.

Thus, the maximum degree of any vertex of $G^{\prime}$ is at most $15+\left\lceil\frac{2 \pi}{\gamma}\right\rceil-1=14+\left\lceil\frac{2 \pi}{\gamma}\right\rceil$. 


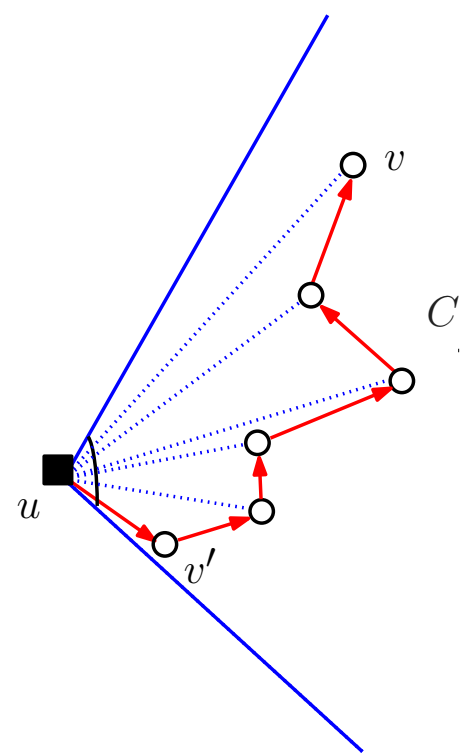

(a) Case 1: All neighbors of $u$ between $v$ and $v^{\prime}$ are unprocessed.

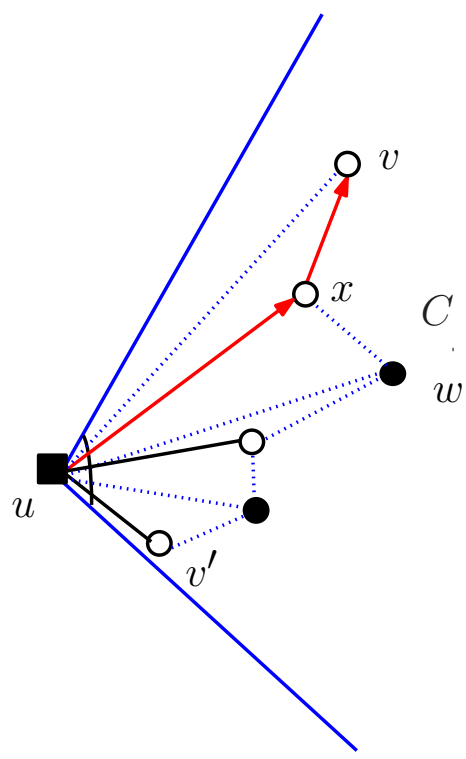

(b) Case 2: At least one neighbor of $u$ between $v$ and $v^{\prime}$ has been processed.

Figure 4: Illustrations of the proof of Lemma 2.

\section{Bounded-degree spanners of the Delaunay triangu- lation}

In this section, we assume that $G=(V, E)$ is the Delaunay triangulation of the point set $V$. Let $G^{\prime}=\left(V, E^{\prime}\right)$ be the output of algorithm BDegSubgraph $(G, \gamma)$. Lemma 3 gives an upper bound on the maximum degree of $G^{\prime}$. In this section, we will prove that $G^{\prime}$ is a spanner of $V$, which will complete the proof of Theorem 1. Our analysis uses the following two lemmas, which state some basic properties of the Delaunay triangulation. These lemmas are illustrated in Figure 5.

Lemma 4. [4] Let $G$ be the Delaunay triangulation of $V$, let $\gamma$ be a real number with $0<\gamma<\pi / 2$, and let $u, v$, and $v^{\prime}$ be three points of $V$, such that $(u, v)$ and $\left(u, v^{\prime}\right)$ are Delaunay edges and $\angle v u v^{\prime} \leq \gamma$. Let $v^{\prime}=s_{1}, s_{2}, \ldots, s_{k-1}, s_{k}=v$ be the Delaunay neighbors of $u$ between $v^{\prime}$ and $v$, sorted in angular order around $u$. Assume that $\left|u v^{\prime}\right| \leq\left|u s_{i}\right|$ for all $i$ with $1 \leq i \leq k$. Finally, let $P_{u v}$ be the path $u, v^{\prime}, s_{2}, s_{3}, \ldots, s_{k-1}, v$ in $G$ between $u$ and $v$. Then, the length of $P_{u v}$ is at most $t_{\gamma}|u v|$, where

$$
t_{\gamma}=\max \left\{\frac{\pi}{2}, 1+\pi \sin \frac{\gamma}{2}\right\} .
$$

Lemma 5. [1] Let $G$ be the Delaunay triangulation of $V$, and let $u, x$, and $y$ be three points of $V$, such that $(u, x)$ and $(u, y)$ are Delaunay edges and $\angle x u y<\pi / 2$. Let $x=$ $s_{1}, s_{2}, \ldots, s_{k-1}, s_{k}=y$ be the Delaunay neighbors of $u$ between $x$ and $y$, sorted in angular 


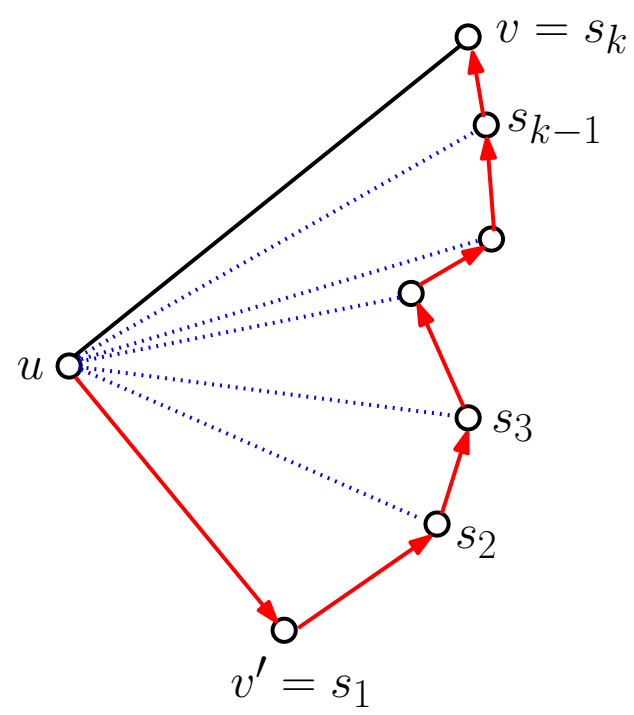

(a) An illustration of Lemma 4.

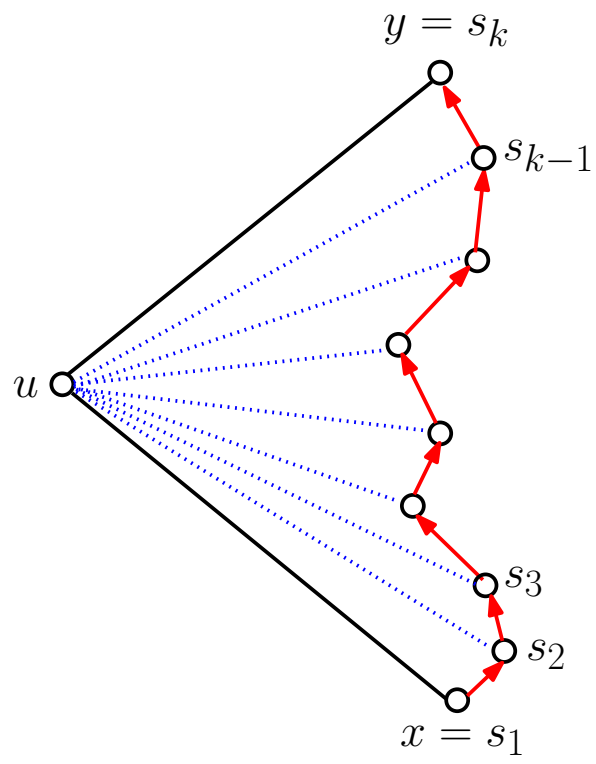

(b) An illustration of Lemma 5 .

Figure 5: Illustrations of Lemma 4 and Lemma 5.

order around $u$. Let $Q_{x y}$ be the path $x, s_{2}, s_{3}, \ldots, s_{k-1}, y$ in $G$ between $x$ and $y$. Then, the length of $Q_{x y}$ is at most

$$
\frac{\pi}{2}(|u x|+|u y|) .
$$

We also need the following two geometric lemmas.

Lemma 6. Given a triangle $\Delta_{a b c}$, if $\angle a b c \leq 2 \pi / 3$ and $|a b| \geq|b c|$, then $|a c| \leq \sqrt{3}|a b|$.

Proof. Refer to Figure 6(a). In triangle $\Delta_{a b c}$, let $\beta=\angle a b c$. By the Law of Cosines, we have

$$
|a c|=\sqrt{|a b|^{2}+|b c|^{2}-2|a b| \cdot|b c| \cos \beta} .
$$

Since $|a b| \geq|b c|$ and $\beta \leq 2 \pi / 3$, we have

$$
|a c| \leq \sqrt{2|a b|^{2}-2|a b| \cdot|b c| \cos (2 \pi / 3)}=\sqrt{2|a b|^{2}+|a b||b c|} \leq \sqrt{3|a b|^{2}}=\sqrt{3}|a b| .
$$

Lemma 7. Let $u, v, w$, and $s$ be four points in the plane, such that $\angle v u w \leq 2 \pi / 3$ and $|u v| \geq|u w|$, and let $C$ be the circle through $u$, $v$, and $w$. Assume that $s$ is contained in $C$. Then,

$$
|u s| \leq 2|u v|
$$




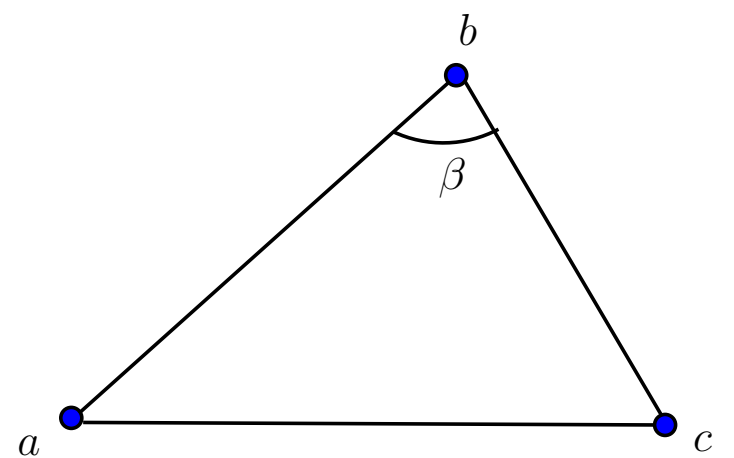

(a) An illustration of Lemma 6.

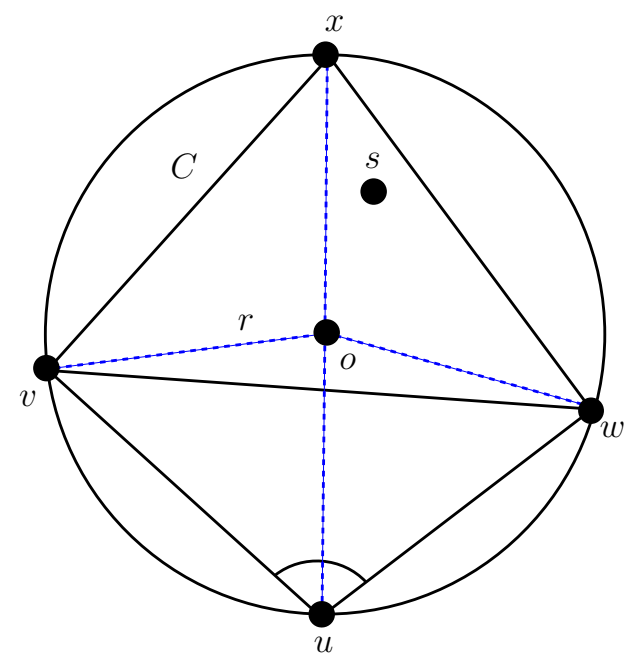

(b) An illustration of Lemma 7.

Figure 6: Illustrations of Lemma 6 and Lemma 7.

Proof. Let $o$ be the center of $C$ and denote the radius of $C$ by $r$; refer to Figure 6(b). Since $s$ is inside $C$ and $u$ is on the boundary of $C$, we have $|u s| \leq 2 r$. Thus, we only need to show that $|u v| \geq r$.

Let $x$ be the point on $C$ for which $|u x|=2 r$. In the right triangles $\Delta_{u v x}$ and $\Delta_{u w x}$, since $|u v| \geq|u w|$, we have $|v x| \leq|w x|$. It follows that $\angle v u x \leq \angle x u w$. Since $\angle v u w=$ $\angle v u x+\angle x u w \leq 2 \pi / 3$, we have $\angle v u x \leq \pi / 3$. In the triangle $\Delta_{v u o}$, we have $|o v|=|o u|=r$ and $\angle v u o=\angle v u x \leq \pi / 3$. It follows that $\angle v o u \geq \pi / 3$ and thus $|u v| \geq|o v|=|o u|=r$.

We can now prove the main result of this section, which completes the proof of Theorem 1.

Lemma 8. Let $G=(V, E)$ be the Delaunay triangulation of $V$, let $\gamma$ be a real number with $0<\gamma \leq 2 \pi / 3$, and let $G^{\prime}=\left(V, E^{\prime}\right)$ be the output of algorithm $\operatorname{BDEGSubGrAPH}(G, \gamma)$. Then, $G^{\prime}$ is a $\frac{4 \pi \sqrt{3}}{9} \cdot t^{\prime}$-spanner of $V$, where

$$
t^{\prime}= \begin{cases}\max \left\{\frac{\pi}{2}, 1+\pi \sin \frac{\gamma}{2}\right\} & \text { if } \gamma<\pi / 2, \\ 2+2 \sqrt{3}+3 \pi / 2+2 \pi \sin \frac{\pi}{12} & \text { if } \pi / 2 \leq \gamma \leq 2 \pi / 3 .\end{cases}
$$

Proof. Recall that $G$ is a $\frac{4 \pi \sqrt{3}}{9}$-spanner of $V$; see Keil and Gutwin [8]. Also, $G^{\prime}$ is a subgraph of $G$. Therefore, it suffices to show that for each edge $(u, v)$ of $E \backslash E^{\prime}$, the graph $G^{\prime}$ contains a path between $u$ and $v$, whose length is at most $t^{\prime}|u v|$.

Throughout the rest of the proof, we fix an edge $(u, v)$ of $E \backslash E^{\prime}$. We may assume without loss of generality that $u$ is processed before $v$. Let $C$ be the cone with apex $u$ and having angle at most $\gamma$ that contains $v$ and that is constructed when vertex $u$ is processed. Let $v^{\prime}$ be the closest Delaunay-neighbor of $u$ that is contained in $C$ and that is unprocessed at the moment when $u$ is processed. Thus, during the processing of $u$, the edge $\left(u, v^{\prime}\right)$ is added to 


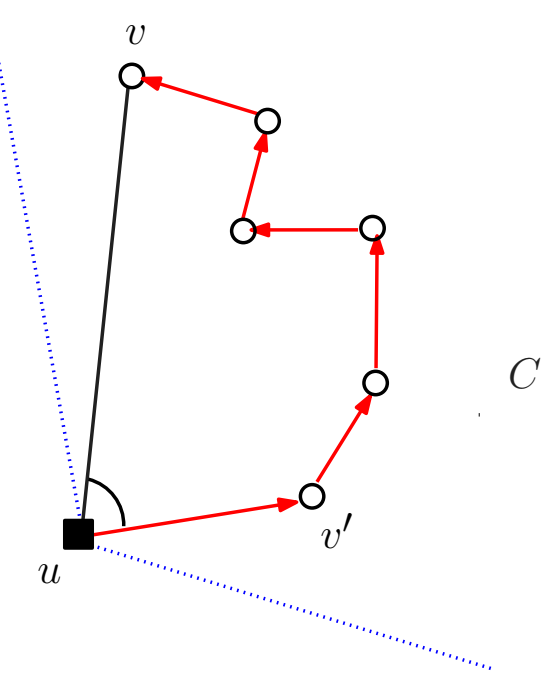

(a) All Delaunay-neighbors of $u$ between $v$ and $v^{\prime}$ are unprocessed.

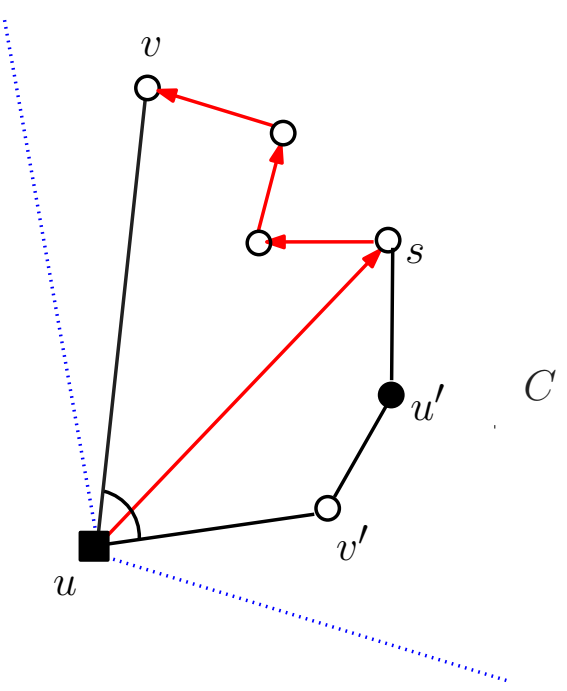

(b) At least one Delaunay-neighbor of $u$ between $v$ and $v^{\prime}$ has been processed.

Figure 7: Case 1 in the proof of Lemma 8.

$G^{\prime}$. We may assume without loss of generality that $\left(u, v^{\prime}\right)$ is clockwise to the right of $(u, v)$. Observe that $\angle v u v^{\prime} \leq \gamma$.

We distinguish three cases.

Case 1: $\angle v u v^{\prime}<\frac{\pi}{2}$.

Consider the path $P_{u v}$ of Lemma 4 between $u$ and $v$ shown as a directed path through vertex $v^{\prime}$ in Figure 7(a). By Lemma 4, the length of this path is at most $t_{\gamma}|u v| \leq t^{\prime}|u v|$.

We first assume that, at the moment when $u$ is processed, all Delaunay-neighbors of $u$ between $v$ and $v^{\prime}$ are unprocessed. Then it follows from algorithm BDEGSuBGRAPH that $P_{u v}$ is a path in $G^{\prime}$.

Now assume that, at the moment when $u$ is processed, at least one Delaunay-neighbor $u^{\prime}$ of $u$ between $v$ and $v^{\prime}$ has already been processed; refer to Figure 7(b). Let $u^{\prime}$ be the first such Delaunay-neighbor in clockwise order from $(u, v)$. Observe that $u^{\prime} \neq v$ and $u^{\prime} \neq v^{\prime}$. Let $s$ be the Delaunay-neighbor of $u$ such that $s$ is between $v$ and $u^{\prime}$ and $\triangle_{u s u^{\prime}}$ is a Delaunaytriangle. Then, by our choice of $u^{\prime}, u^{\prime}$ is processed before $s$. Thus, during the processing of $u^{\prime}$, the algorithm adds the edge $(u, s)$ to $G^{\prime}$, because $G$ is a triangulation and $u$ and $s$ appear consecutively around $u^{\prime}$ in $G$. Let $Q_{s v}$ be the subpath of $P_{u v}$ that starts at $s$ and ends at $v$. Let $Q^{\prime}$ be the path obtained by concatenating the edge $(u, s)$ and the subpath $Q_{s v}$. It follows from algorithm BDEGSubGRAPH that $Q^{\prime}$ is a path in $G^{\prime}$ between $u$ and $v$. By the triangle inequality, the length of $Q^{\prime}$ is at most the length of $P_{u v}$. Thus, the length of $Q^{\prime}$ is at most $t_{\gamma}|u v| \leq t^{\prime}|u v|$

This concludes the analysis of Case 1 . Observe that this case always holds if $\gamma<\pi / 2$.

Case 2: $\angle v u v^{\prime} \geq \frac{\pi}{2}$ and there is at least one Delaunay-neighbor $w$ of $u$ such that $\angle v u w<\frac{\pi}{2}$ and $\angle w u v^{\prime}<\frac{\pi}{2}$. 


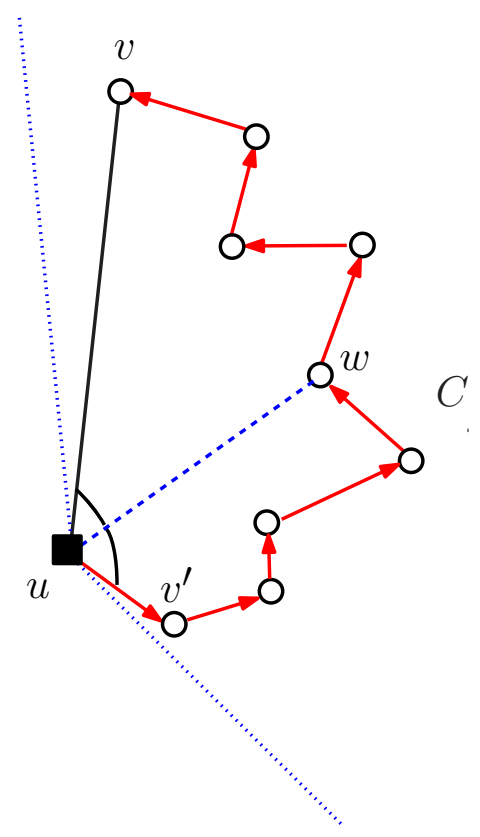

(a) All Delaunay-neighbors of $u$ between $v$ and $v^{\prime}$ are unprocessed.

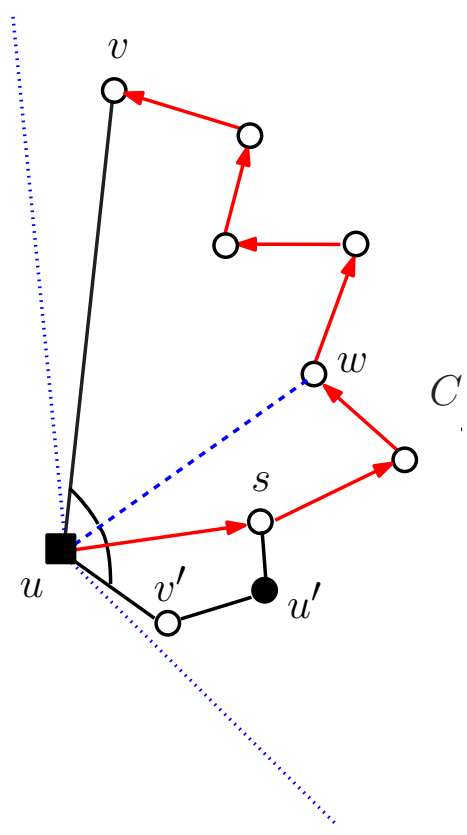

(b) At least one Delaunay-neighbor of $u$ between $v$ and $v^{\prime}$ has been processed.

Figure 8: Case 2 in the proof of Lemma 8.

Let $P_{u w}$ be the path that starts at $u$, follows the edge $\left(u, v^{\prime}\right)$, and then follows the Delaunay-neighbors of $u$ from $v^{\prime}$ to $w$. Let $Q_{w v}$ be the path that starts at $w$, and follows the Delaunay-neighbors of $u$ from $w$ to $v$. Let $P$ be the concatenation of $P_{u w}$ and $Q_{w v}$. (Refer to Figure 8(a)).

The length of $P$ is equal to the sum of the lengths of $P_{u w}$ and $Q_{w v}$. Since $\angle w u v^{\prime}<\frac{\pi}{2}$, it follows from Lemma 4 that the length of $P_{u w}$ is at most $t_{\frac{\pi}{2}}|u w|$, where $t_{\frac{\pi}{2}}=\max \left\{\frac{\pi}{2}, 1+\right.$ $\left.\pi \sin \frac{\pi}{4}\right\}=1+\frac{\pi \sqrt{2}}{2}$. Since $\angle v u w<\frac{\pi}{2}$, it follows from Lemma 5 that the length of $Q_{w v}$ is at most $\frac{\pi}{2}(|u v|+|u w|)$. Thus, the length of $P$ is at most

$$
\frac{\pi}{2}|u v|+\left(t_{\frac{\pi}{2}}+\frac{\pi}{2}\right)|u w|
$$

We prove an upper bound on this quantity in terms of $|u v|$.

Recall that $(u, v),(u, w)$ and $\left(u, v^{\prime}\right)$ are edges in the Delaunay triangulations of $V$. These three edges are also edges in the Delaunay triangulation of the point set $\left\{u, v, v^{\prime}, w\right\}$. It follows that $\Delta_{u v v^{\prime}}$ is not a triangle in the latter triangulation and, therefore, $w$ is in the circle through $u, v$ and $v^{\prime}$. It then follows from Lemma 7 that $|u w| \leq 2|u v|$. Thus, the length of $P$ is at most

$$
\frac{\pi}{2}|u v|+\left(t_{\frac{\pi}{2}}+\frac{\pi}{2}\right) \cdot(2|u v|)=\left(2 t_{\frac{\pi}{2}}+3 \pi / 2\right)|u v| \leq t^{\prime}|u v| .
$$

Assume that, at the moment when $u$ is processed, all Delaunay-neighbors of $u$ between $v$ and $v^{\prime}$ are unprocessed. Then it follows from algorithm BDEGSUBGRAPH that $P$ is a path in 


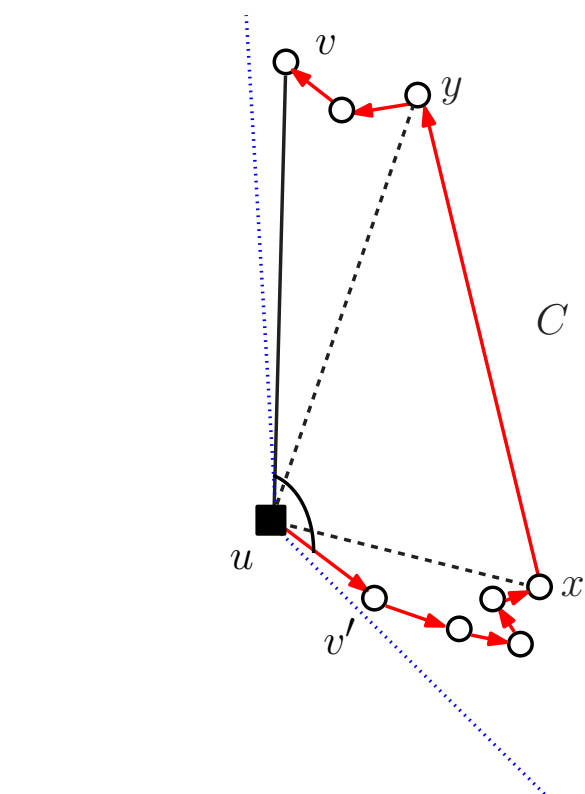

(a) All Delaunay-neighbors of $u$ between $v$ and $v^{\prime}$ are unprocessed.

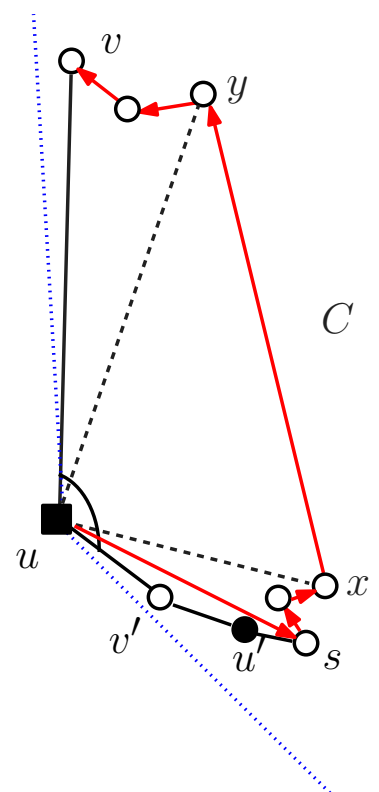

(b) At least one Delaunay-neighbor of $u$ between $v$ and $v^{\prime}$ has been processed.

Figure 9: Case 3 in the proof of Lemma 8.

$G^{\prime}$ between $u$ and $v$. If at least one Delaunay-neighbor $u^{\prime}$ of $u$ between $v$ and $v^{\prime}$ has already been processed (refer to Figure 8(b)), then, by a similar argument as in Case $1, G^{\prime}$ contains a path between $u$ and $v$ which is obtained by shortcutting $P$.

Case 3: $\angle v u v^{\prime} \geq \frac{\pi}{2}$ and there is no Delaunay-neighbor $w$ of $u$ such that $\angle v u w<\frac{\pi}{2}$ and $\angle w u v^{\prime}<\frac{\pi}{2}$.

In this case, there exist two Delaunay-edges $(u, x)$ and $(u, y)$ such that $\angle y u v^{\prime} \geq \frac{\pi}{2}$, $\angle v u x \geq \frac{\pi}{2}$, and $(x, y)$ is a Delaunay-edge (Refer to Figure $9\left(\right.$ a), it may happen that $x=v^{\prime}$ and/or $y=v$.)

Let $P_{u x}$ be the path that starts at $u$, follows the edge $\left(u, v^{\prime}\right)$, and then follows the Delaunay-neighbors of $u$ from $v^{\prime}$ to $x$. Let $Q_{y v}$ be the path that starts at $y$, and follows the Delaunay-neighbors of $u$ from $y$ to $v$. Let $P$ be the concatenation of $P_{u x}$, the edge $(x, y)$, and $Q_{y v}$. Then the length of $P$ is equal to the sum of the lengths of $P_{u x}, Q_{y v}$, and $|x y|$. Since $\angle v u x \geq \frac{\pi}{2}$ and $\angle v u v^{\prime} \leq \frac{2 \pi}{3}$, we have $\angle x u v^{\prime} \leq \frac{\pi}{6}$. Therefore, it follows from Lemma 4 that the length of $P_{u x}$ is at most $t_{\frac{\pi}{6}}|u x|$, where $t_{\frac{\pi}{6}}=\max \left\{\frac{\pi}{2}, 1+\pi \sin \frac{\pi}{12}\right\}=1+\pi \sin \frac{\pi}{12}$. Since $\angle v u v^{\prime} \leq \frac{2 \pi}{3}$ and $\angle y u v^{\prime} \geq \frac{\pi}{2}$, we have $\angle v u y<\frac{\pi}{2}$. Thus, by Lemma 5 , the length of $Q_{y v}$ is at most $\frac{\pi}{2}(|u v|+|u y|)$. Thus, the length of $P$ is at most

$$
t_{\frac{\pi}{6}}|u x|+|x y|+\frac{\pi}{2}(|u v|+|u y|) .
$$

We will prove an upper bound on this quantity in terms of $|u v|$.

Since $\Delta_{u v x}$ is not a triangle in the Delaunay triangulation of $\left\{u, v, v^{\prime}, x\right\}, x$ is in the circle through $u, v$ and $v^{\prime}$. It then follows from Lemma 7 that $|u x| \leq 2|u v|$. By a similar 
argument, $y$ is in the circle through $u, v$ and $v^{\prime}$ and, again by Lemma $7,|u y| \leq 2|u v|$. Let $z=\max (|u x|,|u y|)$. By Lemma 6 , we have $|x y| \leq \sqrt{3} z \leq 2 \sqrt{3}|u v|$. Thus, the length of $P$ is at most

$$
t_{\frac{\pi}{6}}(2|u v|)+2 \sqrt{3}|u v|+\frac{\pi}{2}(3|u v|)=\left(2+2 \sqrt{3}+3 \pi / 2+2 \pi \sin \frac{\pi}{12}\right)|u v|=t^{\prime}|u v| .
$$

If, at the moment when $u$ is processed, all Delaunay-neighbors of $u$ between $v$ and $v^{\prime}$ are unprocessed (see Figure $9(\mathrm{a})$ ), then $P$ is a path in $G^{\prime}$ between $u$ and $v$. Otherwise, at least one Delaunay-neighbor $u^{\prime}$ of $u$ between $v$ and $v^{\prime}$ had already been processed (see Figure $9(\mathrm{~b})$ ). In this case, by a similar argument as in Case $1, G^{\prime}$ contains a path between $u$ and $v$ which is obtained by shortcutting $P$.

\section{Bounded-degree spanners of the unit-disk graph}

Let $V$ be a set of $n$ points in the plane. Let $U D e l$ be the graph with vertex set $V$, and whose edge set is the set of all edges in the Delaunay triangulation of $V$ whose length is at most one. UDel may not be a triangulation, even though it is a plane graph. For simplicity, we assume that $U D e l$ is connected. If $U D e l$ is not connected, then we consider each connected component of $U D e l$ separately. In this section, we show that we can modify algorithm BDEGSUBGRAPH to construct a bounded-degree spanner of the unit-disk graph. The modified algorithm is given in Figure 10. In this algorithm, $N_{G}(v)$ denotes the set of neighbors of the vertex $v$ in a graph $G$, i.e. $N_{G}(v)=\{w \in V:(v, w)$ is an edge in $G\}$.

Observe that in line 25 of this algorithm, edges may be added to the output graph $G^{\prime}$ which are not in $U D e l$; still, each of these edges has length at most one. Thus, the algorithm computes a subgraph $G^{\prime}$ of the unit-disk graph that is not necessarily a subgraph of $U D e l$. As illustrated in Figure 12, edges $\left(s_{1}, s_{2}\right),\left(s_{3}, s_{4}\right)$ and $\left(s_{5}, s_{6}\right)$ which are added to $G^{*}$ in line 7 are not edges of $U D e l$. Out of these three edges, only $\left(s_{3}, s_{4}\right)$ is added to $G^{\prime}$.

We assume that the Delaunay triangulation is stored in a doubly-connected edge list. Then, for any vertex $v$, the time spent for adding edges in line 7 is proportional to the degree of $v$ in the Delaunay triangulation of $V$. We can also obtain the vertices in $N_{G^{*}}(v)$, sorted in angular order around $v$, in time proportional to the degree of $v$ in $G^{*}$. It will be shown in Lemma 9 that $G^{*}$ is a plane graph and thus contains $O(n)$ edges. These observations, together with Lemma 1, imply that the running time of algorithm BDEGUDEL is $O(n)$.

In Lemma 9 and 10 we show that, when $0<\gamma \leq \pi / 3$, the graph $G^{\prime}$ is a plane boundeddegree spanner of the unit-disk graph of $V$.

Lemma 9. Let $G=(V, E)$ be the Delaunay triangulation of $V$, and let $\gamma$ be a real number with $0<\gamma \leq \pi / 3$. Let $U D G(V)$ be the unit-disk graph on $V$. Let $G^{\prime}$ be the graph that is returned by algorithm $\operatorname{BDEGUDeL}(G, \gamma)$. Then, $G^{\prime}$ is a plane subgraph of $U D G(V)$ and the maximum degree of $G^{\prime}$ is at most $14+\left\lceil\frac{2 \pi}{\gamma}\right\rceil$. 


\section{Algorithm BDegUDeL $(G, \gamma)$}

Input: The Delaunay triangulation $G=(V, E)$ whose vertex set $V$ is a set of $n$ points in the plane, and a real number $\gamma$ with $0<\gamma<\pi / 3$.

1. let $U D e l$ be the subgraph of $G$ consisting of all edges of length at most one;

2. let $G^{*}=U D e l$;

3. for each $v \in V$

4. $\quad$ do let $u_{0}, u_{1}, \cdots, u_{d-1}$ be the vertices in $N_{U D e l}(v)$, ordered in clockwise order around $v$, where $d$ is the degree of $v$ in $U D e l$;

5. $\quad$ for $k=0$ to $d-1$

6. $\quad$ if $\left(v, u_{k}, u_{(k+1) \bmod d}\right)$ is not a triangle in $G$ and $\left|u_{k} u_{(k+1) \bmod d}\right| \leq 1$

7. $\quad$ then add the edge $\left(u_{k}, u_{(k+1) \bmod d}\right)$ to $G^{*}$;

8. compute a low-degree numbering $\left(v_{1}, v_{2}, \cdots, v_{n}\right)$ of $G^{*}$;

9. label each vertex of $V$ as "unprocessed";

10. $E^{\prime}=\emptyset$;

11. for $i=n$ downto 1

12. if $v_{i}$ has "unprocessed" Delaunay neighbors in $U$ Del (i.e. these neighbors were not added in steps 5-7)

13. then compute the closest "unprocessed" Delaunay neighbor $x$ of $v_{i}$ in UDel; 14. divide the plane into cones $C_{1}, \ldots, C_{\lceil 2 \pi / \gamma\rceil}$ with apex $v_{i}$ and angle at most $\gamma$ such that the segment $v_{i} x$ is on the boundary between $C_{1}$ and $\mathrm{C}_{2}$;

15. $\quad$ add the edge $\left(v_{i}, x\right)$ to $E^{\prime}$

16. $\quad$ for each cone $C \notin\left\{C_{1}, C_{2}\right\}$

17. do compute the closest "unprocessed" Delaunay neighbor $w$ in $C \cap$ $N_{U D e l}\left(v_{i}\right)$;

$18 . \quad$ if $w$ exists

19.

then add the edge $\left(v_{i}, w\right)$ to $E^{\prime}$

20. let $w_{0}, w_{1}, \cdots, w_{d-1}$ be the vertices in $N_{G^{*}}\left(v_{i}\right)$, ordered in clockwise order around $v_{i}$, where $d$ is the degree of $v_{i}$ in $G^{*}$;

21. $\quad$ for $k=0$ to $d-1$

22. $\quad$ if $w_{k}$ and $w_{(k+1) \bmod d}$ are both "unprocessed", $\left(w_{k}, w_{(k+1) \bmod d}\right) \in G^{*}$

23. $\quad$ and $\left|w_{k} w_{(k+1) \bmod d}\right| \leq 1$

24. $\quad$ then add the edge $\left(w_{k}, w_{(k+1) \bmod d}\right)$ to $E^{\prime}$;

25. label $v_{i}$ as "processed";

26. return the graph $G^{\prime}=\left(V, E^{\prime}\right)$

Figure 10: The algorithm that computes a bounded-degree spanner of the unit-disk graph. 


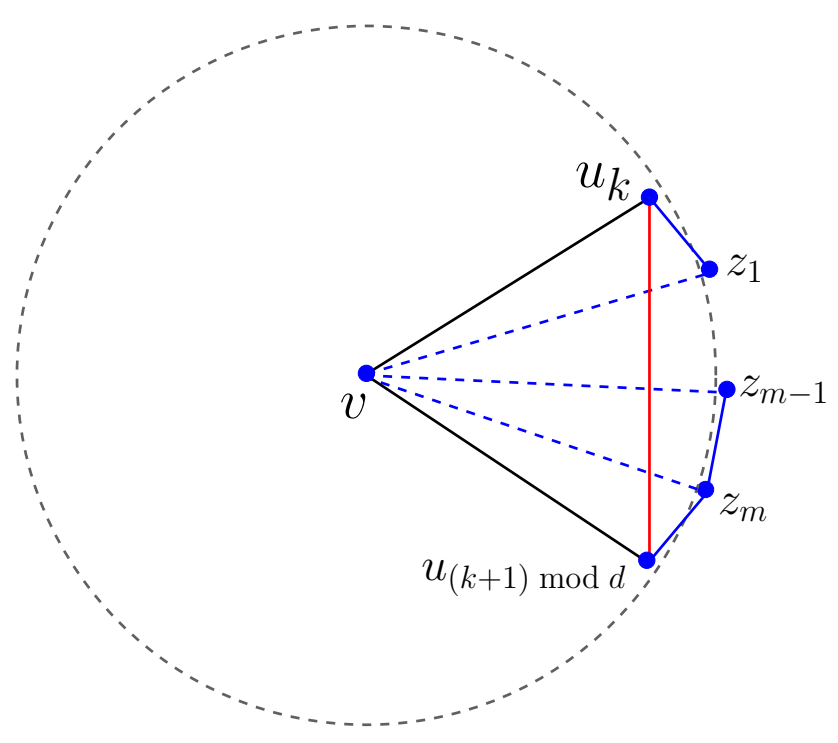

Figure 11: An illustration of the proof of Lemma 9.

Proof. Since $G$ is the Delaunay triangulation of $V, G$ is plane. Therefore, the only possible intersections in $G^{\prime}$ are caused by the edges added by algorithm $\operatorname{BDEGUDeL}(G, \gamma)$ but that are not in $G$. For each edge $(u, v)$ of $G^{\prime}$ that is not in $G$, we need to show that $(u, v)$ will not introduce an edge crossing. Note that edge $(u, v)$ can only be added to $G^{\prime}$ in line 25 of algorithm BDegUDed $(G, \gamma)$. This can happen only if it has been added to $G^{*}$ in line 7 .

In line 7 , edge $\left(u_{k}, u_{(k+1) \bmod d}\right)$ is added to $G^{*}$, which may be kept in the final graph $G^{\prime}$, provided that $\left(v, u_{k}, u_{(k+1) \bmod d}\right)$ is not a triangle in $G$ and $\left|u_{k} u_{(k+1) \bmod d}\right| \leq 1$. Since $\left(v, u_{k}, u_{(k+1) \bmod d}\right)$ is not a Delaunay triangle and $\left(v, u_{k}\right),\left(v, u_{(k+1) \bmod d}\right)$ are Delaunay edges of $G$, there must be some vertices $z_{1}, z_{2}, \cdots, z_{m}, m \geq 1$, ordered in clockwise order around $v$ and bounded by $\left(v, u_{k}\right)$ and $\left(v, u_{(k+1) \bmod d}\right)$ such that $\left(v, z_{j-1}, z_{j}\right), 1 \leq j \leq m+1$, is a Delaunay triangle of $G$, where $z_{0}=u_{k}$ and $z_{m+1}=u_{(k+1) \bmod d}$ (see Figure 11). Since $u_{k}$ and $u_{(k+1) \bmod d}$ are consecutive neighbors of $v$ in $U$ Del, we have that $z_{j}, 1 \leq j \leq m$, must be outside of the unit circle of $v$, i.e., $\left|v z_{j}\right|>1,1 \leq j \leq m$. Since $\left|v z_{j}\right|>1$ and the length of every edge of $G^{\prime}$ is at most $1,\left(v, z_{j}\right) \notin G^{\prime}$. Since $\left|v u_{k}\right|,\left|v u_{(k+1) \bmod d}\right| \leq 1,\left|v z_{j}\right|>1$ and $u_{k}, z_{1}, \cdots, z_{m}, u_{(k+1) \text { mod } d}$ are consecutive Delaunay neighbors of $v$ ordered in clockwise order, $\left(u_{k}, u_{(k+1) \bmod d}\right)$ is only intersected by the Delaunay edges $\left(v, z_{j}\right), 1 \leq j \leq m$, between edges $\left(v, u_{k}\right)$ and $\left(v, u_{(k+1) \bmod d}\right)$. Since $\left(v, z_{j}\right) \notin G^{\prime},\left(u_{k}, u_{(k+1) \bmod d}\right)$ does not introduce any edge crossing in $G^{\prime}$ and thus planarity holds.

Let $u$ be an arbitrary element of $V$. Similar to Lemma 1 , before $u$ is processed, it has at most 5 processed neighbors, say $w_{1}, w_{2}, \cdots, w_{k}$, where $k \leq 5$. In the worst case, each of its processed neighbors $w_{j}$ can increase the degree of $u$ by 3 . Hence, before $u$ is processed, the degree of $u$ (in $G^{\prime}$ ) is at most 15 .

During the processing of $u$, the algorithm divides the plane into $\left\lceil\frac{2 \pi}{\alpha}\right\rceil$ cones. For each cone, the algorithm adds one edge from $u$ to its closest unprocessed neighbor. However, two of these cones $\left(C_{1}\right.$ and $C_{2}$ in the algorithm) share an edge. Thus, during the processing of 


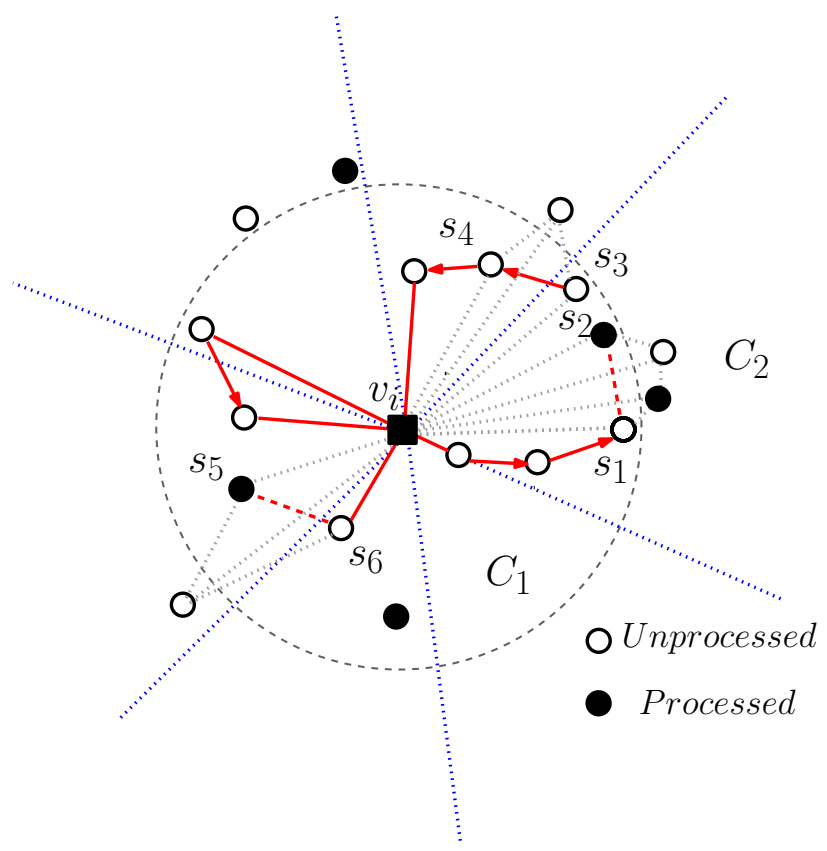

Figure 12: An illustration of algorithm $\operatorname{BDEGUDEL}(G, \gamma)$, for $\gamma=\pi / 3$, when processing vertex $v_{i}$. The figure shows $v_{i}$, all vertices in $N_{G^{*}}\left(v_{i}\right)$ and all Delaunay neighbors of $v_{i}$ whose distance from $v_{i}$ is greater than one. When processing $v_{i}$, the algorithm adds the dashed edges to the graph $G^{*}$ and the solid and arrowed edges to the graph $G^{\prime}$.

$u$, the degree of $u$ (in $G^{\prime}$ ) is increased by at most $\left\lceil\frac{2 \pi}{\gamma}\right\rceil-1$.

After $u$ has been processed, the degree of $u$ does not change.

Thus, the maximum degree of any vertex of $G^{\prime}$ is at most $15+\left\lceil\frac{2 \pi}{\gamma}\right\rceil-1=14+\left\lceil\frac{2 \pi}{\gamma}\right\rceil$.

We now prove that the output of algorithm $\operatorname{BDEGUDeL}(G, \gamma)$ is a spanner of the unitdisk graph. This will complete the proof of Theorem 2

Lemma 10. Let $G=(V, E)$ be the Delaunay triangulation of $V$, and let $\gamma$ be a real number with $0<\gamma \leq \pi / 3$. Let $U D G(V)$ be the unit-disk graph on $V$. Let $G^{\prime}$ be the graph that is returned by algorithm $\operatorname{BDEGUDeL}(G, \gamma)$. Then, $G^{\prime}$ is a $\frac{4 \pi \sqrt{3}}{9} \cdot t_{\gamma}$-spanner of $U D G(V)$, where

$$
t_{\gamma}=\max \left\{\frac{\pi}{2}, 1+\pi \sin \frac{\gamma}{2}\right\} .
$$

Proof. The proof is similar to the proof of Lemma 8. Recall that $U D e l$ is a $\frac{4 \pi \sqrt{3}}{9}$-spanner of $U D G(V)$; see Bose et al. [3]. Therefore, it suffices to show that for each edge $(u, v)$ of $U D e l$ that is not in $G^{\prime}$, the graph $G^{\prime}$ contains a path between $u$ and $v$, whose length is at most $t_{\gamma}|u v|$.

Throughout the remainder of the proof, we fix an edge $(u, v)$ of $U$ Del that is not in $G^{\prime}$. We may assume without loss of generality that $u$ is processed before $v$. Let $C$ be the cone 


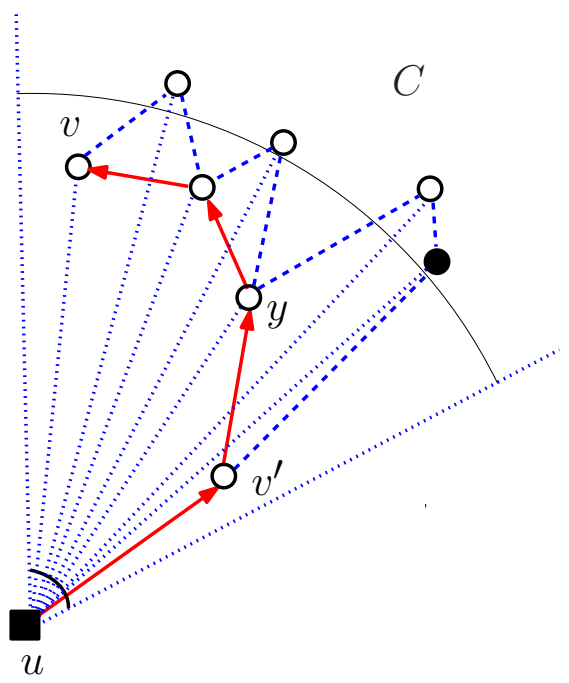

(a) All the neighbors of $u$ between $v$ and $v^{\prime}$ are unprocessed.

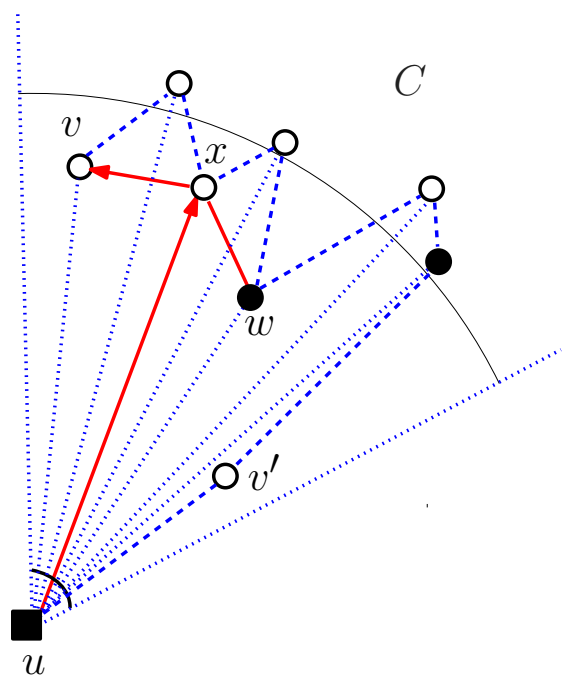

(b) At least one neighbor of $u$ between $v$ and $v^{\prime}$ has been processed.

Figure 13: Illustrations of the proof of Lemma 10.

constructed when vertex $u$ is processed with apex $u$ and angle at most $\gamma$ that contains $v$. Let $v^{\prime}$ be the closest Delaunay neighbor of $u$ in $U D e l$ that is contained in $C$ and that is unprocessed at the moment when $u$ is processed. Thus, during the processing of $u$, the edge $\left(u, v^{\prime}\right)$ is added to $G^{\prime}$. We may assume without loss of generality that $\left(u, v^{\prime}\right)$ is clockwise to the right of $(u, v)$. Observe that $\angle v u v^{\prime} \leq \gamma$.

Consider the path $P_{u v}$ in the Delaunay triangulation of $V$ of Lemma 4 between $u$ and $v$ shown as a dashed path through vertex $v^{\prime}$ in Figure 13(a). By Lemma 4, the length of this path is at most $t_{\gamma}|u v|$, where $t_{\gamma}=\max \left\{\frac{\pi}{2}, 1+\pi \sin \frac{\gamma}{2}\right\}$.

For every maximal subpath of $P_{u v}$ whose internal vertices have distance more than one from $u$, the algorithm has added a shortcut edge connecting the endpoints of this subpath (see line 7 of the algorithm). By replacing each such maximal subpath by its shortcut edge, we obtain a path $Q_{u v}$ in $G^{*}$ between $u$ and $v$. $\left(Q_{u v}\right.$ is shown as a solid directed path in Figure 13(a).) By the triangle inequality, the length of $Q_{u v}$ is at most the length of $P_{u v}$. Therefore, $Q_{u v}$ has length at most $t_{\gamma}|u v|$.

We need to show that there is a path $Q_{u v}^{\prime}$ in $G^{\prime}$ between $u$ and $v$ through all the neighbors of $u$ between $v$ and $v^{\prime}$ in $G^{*}$. In fact, we will show that $Q_{u v}^{\prime}=Q_{u v}$. To show $Q_{u v}^{\prime}$ and $Q_{u v}$ are exactly same, we only need to show that all the vertices in $Q_{u v}^{\prime}$ are Delaunay neighbors of $u$ in $U D e l$. Assume that there is a vertex $y \in Q_{u v}$, which is not a Delaunay neighbor of $u$. This means that edge $(u, y)$ was added to $G^{*}$ in step 7 of algorithm BDEGUDEL. This implies that there is a Delaunay edge $\left(s, s^{\prime}\right)$ that intersects the edge $(u, y)$, where $s$ and $s^{\prime}$ are two consecutive Delaunay neighbors of $u$ in $G,|u s| \leq 1,\left|u s^{\prime}\right| \leq 1$ and $\left|s s^{\prime}\right|>1$. Thus, the angle $\angle s u s^{\prime}$ is larger than $\pi / 3$ and $C$ contains at most one of $s$ and $s^{\prime}$. Without loss of generality, assume that $s^{\prime} \notin C$; refer to Figure 14 . Notice that $\left(u, v^{\prime}\right)$ is clockwise to the right of $\left(u, s^{\prime}\right)$. Therefore, we have $v^{\prime} \notin C$, which contradicts the fact that $v^{\prime} \in C$. Therefore, all 


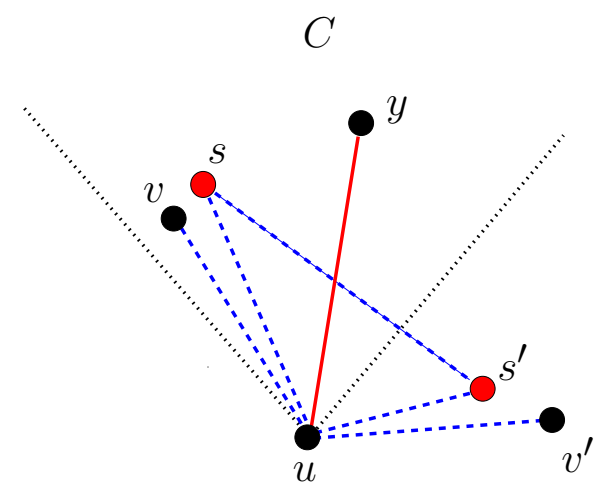

Figure 14: An illustration of the proof of Lemma 10.

the vertices in $Q_{u v}^{\prime}$ are Delaunay neighbors of $u$ in $U D e l$ and $Q_{u v}^{\prime}=Q_{u v}$.

We first assume that, at the moment when $u$ is processed, all vertices of $Q_{u v}$ are unprocessed. An analysis identical to the one in Case 1 in the proof of Lemma 8 shows that $Q_{u v}$ is a path in $G^{\prime}$. Now assume that, at the moment when $u$ is processed, at least one neighbor $w$ of $u$ between $v$ and $v^{\prime}$ has already been processed; refer to Figure 13(b). Let $w$ be the first such neighbor in clockwise order from $(u, v)$. Observe that $w \neq v$ and $w \neq v^{\prime}$. Let $x$ be the first vertex on the subpath of $Q_{u v}$ between $w$ and $v$. Then, by our choice of $w$, it is processed before $x$. Thus, during the processing of $w$, if edge $(w, x)$ is a Delaunay edge of $U D e l$, the algorithm adds the edge $(u, x)$ to $G^{\prime}$. If edge $(w, x)$ is a newly added edge in line 7 , since $(u, w)$ and $(u, x)$ are Delaunay edges of $U$ Del and $u$ is unprocessed during the processing of $w$, the algorithm adds the edge $(u, x)$ to $G^{\prime}$ too. Let $Q$ be the subpath of $Q_{u v}$ that starts at $x$ and ends at $v$. Let $Q^{\prime}$ be the path obtained by concatenating the edge $(u, x)$ and the subpath $Q$. It follows from algorithm BDEGUDEL that $Q^{\prime}$ is a path in $G^{\prime}$ between $u$ and $v$. By the triangle inequality, the length of $Q^{\prime}$ is at most the length of $Q_{u v}$. Thus, the length of $Q^{\prime}$ is at most $t_{\gamma}|u v|$.

\section{Bounded-degree spanners of a diamond triangula- tion}

Let $V$ be a set of $n$ points in the plane, let $\alpha$ be a real number with $0<\alpha<\frac{\pi}{2}$, and let $G=(V, E)$ be a triangulation that satisfies the $\alpha$-diamond property; see Section 1 for the definition of this property. In this section, we complete the proof of Theorem 3, by showing that the output $G^{\prime}=\left(V, E^{\prime}\right)$ of algorithm $\operatorname{BDEGSubGrAPH}(G, \alpha)$ is a $t$-spanner, for some value $t$ that only depends on $\alpha$. The key to the proof is the following lemma which generalizes Lemma 4.

Lemma 11. Let $G=(V, E)$ be a triangulation of the point set $V$, and let $\alpha$ be a real number with $0<\alpha<\frac{\pi}{2}$, such that $G$ satisfies the $\alpha$-diamond property. Let $u, v$ and $v^{\prime}$ be three points 
of $V$, such that $(u, v)$ and $\left(u, v^{\prime}\right)$ are edges of $G$ and $\angle v u v^{\prime} \leq \alpha$. Let $v^{\prime}=s_{1}, s_{2}, \cdots, s_{k}=v$ be the neighbors of $u$ in $G$ between $v^{\prime}$ and $v$, sorted in angular order around $u$. Assume that $\left|u v^{\prime}\right| \leq\left|u s_{i}\right|$ for all $i$ with $1 \leq i \leq k$. Let $P_{u v}$ be the path $u, v^{\prime}, s_{2}, s_{3}, \cdots, s_{k-1}, v$. Then, $P_{u v}$ is a path in $G$ between $u$ and $v$, whose length is at most $t_{\alpha}^{\prime}|u v|$, where

$$
t_{\alpha}^{\prime}=1+\frac{2(\pi-\alpha)}{\alpha \sin \frac{\alpha}{4}} \cdot \max \left\{1,2 \sin \frac{\alpha}{2}\right\} .
$$

Proof. Let $Q$ be the path $v^{\prime}=s_{1}, s_{2}, s_{3}, \cdots, s_{k-1}, s_{k}=v$. Let $R$ be the shortest path between $v^{\prime}$ and $v$ that is completely contained in the polygon with vertices $u, v^{\prime}, s_{2}, \ldots, s_{k-1}, v$. (In Figure $15(\mathrm{a}), R=\left(v^{\prime}, s_{3}, s_{6}, v\right)$.) Consider any edge $\left(s_{i}, s_{j}\right)$ of $R$, and let $Q^{\prime}=\left(s_{i}, s_{i+1}, \ldots, s_{j}\right)$ be the corresponding subpath of $Q$. Observe that $Q^{\prime}$ is completely on one side of the line segment $s_{i} s_{j}$. (An example is the subpath $\left(s_{3}, s_{4}, s_{5}, s_{6}\right)$ in Figure 15(a).)

Consider an arbitrary vertex $s_{m}$ of $Q^{\prime}$ with $i<m<j$. Since $\left(u, s_{m}\right)$ is an edge in $G$, and since $G$ satisfies the $\alpha$-diamond property, at least one of the two isosceles triangles $\Delta_{1}$ and $\Delta_{2}$ with base $\left(u, s_{m}\right)$ and base angle $\alpha$ does not contain any point of $V$ in its interior. Let $a_{1}$ and $a_{2}$ be the two apices of these triangles; see Figure 15(b). We may assume without loss of generality that the triangle with apex $a_{1}$ is empty. We claim that $a_{1}$ and $u$ are on the same side of the line through $s_{i}$ and $s_{j}$. Indeed, assume that $a_{1}$ is "below" $\left(s_{i}, s_{j}\right)$, then, since $\angle a_{1} u s_{m}=\alpha$, the triangle $\triangle_{u a_{1} s_{m}}$ must contain the vertex $s_{i}$, which is a contradiction.

Thus, for each vertex $s_{m}$ on $Q^{\prime}$, with $i<m<j$, the apex of the empty $\alpha$-triangle of the edge $\left(u, s_{m}\right)$ is on the same side of the line through $s_{i} s_{j}$ as $u$. $Q^{\prime}$ is called a one-sided path.

Das and Joseph [6] and Lee [2] showed that the length of a one-sided path from $a$ to $b$ can be bounded in terms of $|a b|$ in the following way: Let $L$ be a one-sided path from $a$ to $b$ in $H$, where $H$ is a triangulation satisfying the $\alpha$-diamond property. Then the length of $L$ is at most $c_{\alpha} \cdot|a b|$, where $c_{\alpha}=\frac{2(\pi-\alpha)}{\alpha \cdot \sin (\alpha / 4)}$.

Hence, the length of $Q$ is at most $c_{\alpha}$ times the length of $R$. We now prove an upper bound on the length of $R$.

Let $w$ be the point on the edge $(u, v)$ such that $\left|u v^{\prime}\right|=|u w|$. We define $\ell^{\prime}=\left|u v^{\prime}\right|$ and $\ell=|u v|$, and observe that $\ell \geq \ell^{\prime}$. Since the length of each edge $\left(u, s_{m}\right), 1 \leq m \leq k$, is at least $\ell^{\prime}$, no point can be inside the triangle $\Delta_{u v^{\prime} w}$. Since $R$ is the shortest path between $v^{\prime}$ and $v$ that is completely contained in the polygon with vertices $u, v^{\prime}, s_{2}, \ldots, s_{k-1}, v$, by convexity, each point in $R$ must be inside the triangle $\Delta_{u v^{\prime} v}$. Thus, the path $R$ is contained in the triangle $\Delta_{v^{\prime} v w}$. Therefore, by convexity, the length of $R$ is at most $|v w|+\left|w v^{\prime}\right|$. Denote $\angle v u v^{\prime}$ by $\theta$ and observe that $\theta \leq \alpha$. Then, we have

$$
\begin{aligned}
|v w|+\left|w v^{\prime}\right| & =\ell-\ell^{\prime}+2 \ell^{\prime} \sin \frac{\theta}{2} \\
& \leq \ell-\ell^{\prime}+2 \ell^{\prime} \sin \frac{\alpha}{2} \\
& =\ell-\left(1-2 \sin \frac{\alpha}{2}\right) \ell^{\prime} .
\end{aligned}
$$

Thus, if $1-2 \sin \frac{\alpha}{2} \geq 0$, then $|v w|+\left|w v^{\prime}\right| \leq \ell^{\prime}=|u v|$. On the other hand, if $1-2 \sin \frac{\alpha}{2}<0$, then

$$
|v w|+\left|w v^{\prime}\right| \leq \ell+\left(2 \sin \frac{\alpha}{2}-1\right) \ell^{\prime} \leq \ell+\left(2 \sin \frac{\alpha}{2}-1\right) \ell=2 \sin \frac{\alpha}{2}|u v| .
$$




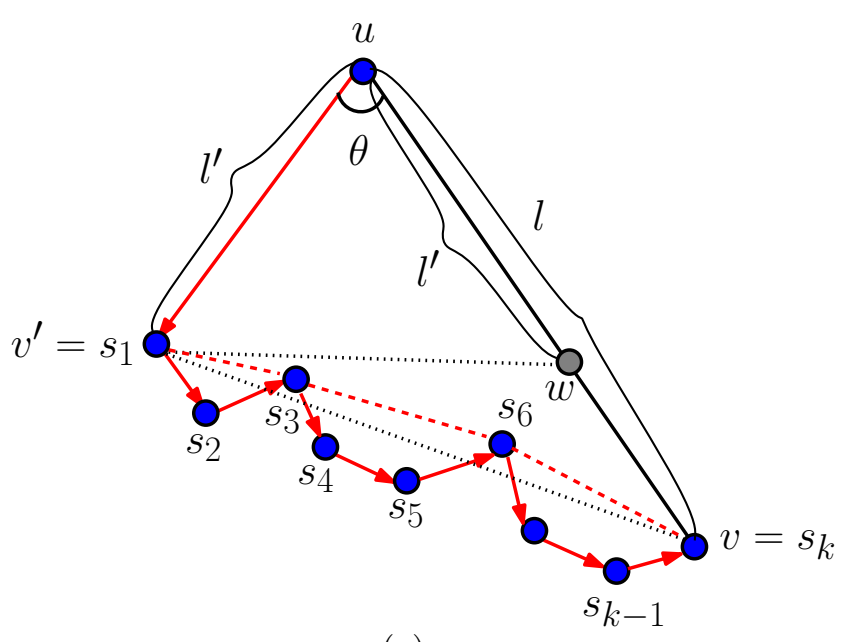

(a)

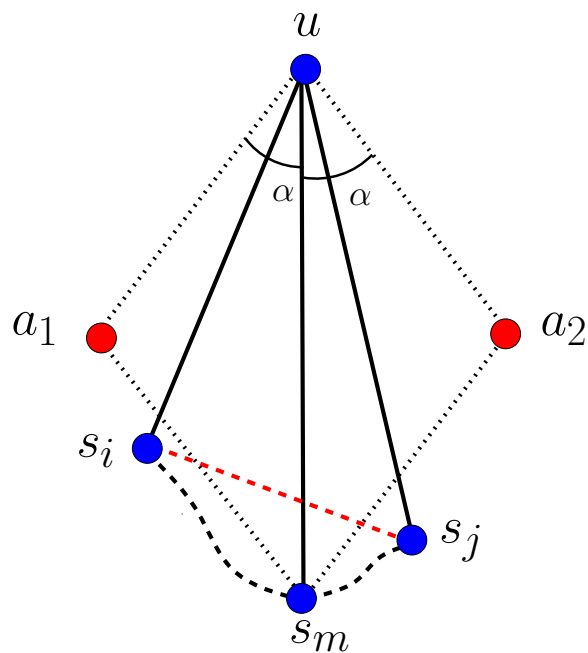

(b)

Figure 15: An illustration of Lemma 11.

This shows that,

$$
|v w|+\left|w v^{\prime}\right| \leq \max \left\{1,2 \sin \frac{\alpha}{2}\right\} \cdot|u v| .
$$

We conclude that the length of the path $P_{u v}$ in the lemma is at most $\left|u v^{\prime}\right|$ plus $c_{\alpha}$ times the length of $R$, which in turn is at most $|u v|+c_{\alpha} \cdot \max \left\{1,2 \sin \frac{\alpha}{2}\right\}|u v|$.

We are now able to prove that algorithm BDEGSubGraph $(G, \alpha)$ computes a spanner of the diamond triangulation $G$ and thereby completing the proof of Theorem 3.

Lemma 12. Let $G=(V, E)$ be a triangulation and let $\alpha$ be a real number with $0<\alpha<\frac{\pi}{2}$, such that $G$ satisfies the $\alpha$-diamond property. Let $G^{\prime}=\left(V, E^{\prime}\right)$ be the output of algorithm $\operatorname{BDegSubgraph}(G, \alpha)$. Then, $G^{\prime}$ is a t-spanner of $V$, where

$$
t=t_{\alpha}^{\prime} \cdot \frac{8(\pi-\alpha)^{2}}{\alpha^{2} \sin ^{2} \frac{\alpha}{4}}
$$

and $t_{\alpha}^{\prime}$ is given in Lemma 11.

Proof. Let $(u, v)$ be an arbitrary edge of $E \backslash E^{\prime}$. Using the same proof technique as in Theorem 1, and using Lemma 11, it can be shown that $G^{\prime}$ contains a path between $u$ and $v$ whose length is at most $t_{\alpha}^{\prime}|u v|$. (Since $0<\alpha<\frac{\pi}{2}$, only Case 1 in the proof of Theorem 1 needs to be considered.) Since Das and Joseph [6] and Lee [2] have shown that $G$ is a $t^{\prime}$-spanner of $V$, for $t^{\prime}=\frac{8(\pi-\alpha)^{2}}{\alpha^{2} \sin ^{2} \frac{\alpha}{4}}$, the lemma follows.

\section{Concluding remarks}

We have shown that the Delaunay triangulation and, in fact, any triangulation satisfying the diamond property, contains a $t$-spanner of bounded degree, for some constant $t$. The 
smallest degree bound that we obtained is 17 (see Theorem 1). We leave open the problem of further reducing the degree. To be more precise, we pose the following open problems:

1. What is the smallest integer $d$, such that there exists a constant $t$ (depending on $d$ ), such that the Delaunay triangulation of any point set $V$, or any triangulation satisfying the diamond property, contains a subgraph which is a $t$-spanner of $V$ ?

2. What is the smallest integer $d$, such that there exists a constant $t$ (depending on $d$ ), such that for every point set $V$, a plane $t$-spanner of $V$ exists?

We pose the same open problems for spanners of the unit-disk graph.

\section{References}

[1] P. Bose, J. Gudmundsson, and M. Smid. Constructing plane spanners of bounded degree and low weight. Algorithmica, 42:249-264, 2005.

[2] P. Bose, A. Lee, and M. Smid. On generalized diamond spanners. In Proceedings of the 10th Workshop on Algorithms and Data Structures (WADS), volume 4619 of Lecture Notes in Computer Science, pages 325-336, Berlin, 2007. Springer-Verlag.

[3] P. Bose, A. Maheshwari, G. Narasimhan, M. Smid, and N. Zeh. Approximating geometric bottleneck shortest paths. Computational Geometry: Theory and Applications, 29:233-249, 2004.

[4] P. Bose and P. Morin. Online routing in triangulations. SIAM Journal on Computing, 33:937-951, 2004.

[5] L. P. Chew. There are planar graphs almost as good as the complete graph. Journal of Computer and System Sciences, 39:205-219, 1989.

[6] G. Das and D. Joseph. Which triangulations approximate the complete graph? In Proceedings of the International Symposium on Optimal Algorithms, volume 401 of Lecture Notes in Computer Science, pages 168-192, Berlin, 1989. Springer-Verlag.

[7] D. P. Dobkin, S. J. Friedman, and K. J. Supowit. Delaunay graphs are almost as good as complete graphs. Discrete \& Computational Geometry, 5:399-407, 1990.

[8] J. M. Keil and C. A. Gutwin. Classes of graphs which approximate the complete Euclidean graph. Discrete \&6 Computational Geometry, 7:13-28, 1992.

[9] X.-Y. Li and Y. Wang. Efficient construction of low weighted bounded degree planar spanner. International Journal of Computational Geometry 85 Applications, 14:69-84, 2004.

[10] G. Narasimhan and M. Smid. Geometric Spanner Networks. Cambridge University Press, Cambridge, UK, 2007. 